\title{
Efficient inhibition of duck hepatitis B virus DNA by the CRISPR/Cas9 system
}

\author{
QINGFEN ZHENG $^{1-3^{*}}$, LI BAI $^{1 *}$, SUJUN ZHENG $^{1 *}$, MEI LIU $^{1}$, JINYAN ZHANG $^{1}$, TING WANG $^{1}$, \\ ZHONGWEI XU ${ }^{4}$, YU CHEN ${ }^{1}$, JIANSHENG $\mathrm{LI}^{2}$ and ZHONGPING DUAN ${ }^{1}$ \\ ${ }^{1}$ Artificial Liver Center, Beijing Youan Hospital, Capital Medical University, Beijing $100069 ;{ }^{2}$ Department of \\ Gastroenterology, The First Affiliated Hospital of Zhengzhou University, Zhengzhou, Henan 450052; ${ }^{3}$ Department of \\ Gastroenterology, The First Affiliated Hospital of Xinxiang Medical College, Weihui, Henan 410781, P.R. China; \\ ${ }^{4}$ Department of Gastroenterology, Pennsylvania Hospital, University of Pennsylvania, Philadelphia, PA 19104, USA
}

Received May 1, 2016; Accepted March 28, 2017

DOI: $10.3892 / \mathrm{mmr} .2017 .7518$

\begin{abstract}
Current therapeutic strategies cannot eradicate hepatitis B virus covalently closed circular DNA (HBV cccDNA), which accounts for the persistence of HBV infection. Very recently, the clustered regularly interspaced short palindromic repeat (CRISPR)/CRISPR-associated protein 9 (Cas9) system has been used as an efficient and powerful tool for viral genome editing. Given that the primary duck hepatocyte $(\mathrm{PDH})$ infected with duck hepatitis $\mathrm{B}$ virus (DHBV) has been widely used to study human HBV infection in vitro, the present study aimed to demonstrate the targeted inhibition of DHBV DNA, especially cccDNA, by the CRISPR/Cas9 system using this model. We designed six single-guide RNAs (sgRNA1-6) targeting the DHBV genome. The sgRNA/Cas9 plasmid was transfected into DHBV-infected PDHs, and then DHBV total DNA (in culture medium and PDHs) and cccDNA were quantified by reverse transcription-quantitative
\end{abstract}

Correspondence to: Professor Zhongping Duan, Artificial Liver Center, Beijing Youan Hospital, Capital Medical University, 8 Xitoutiao Road, Fengtai, Beijing 100069, P.R. China

E-mail: duan2517@163.com

Dr Jiansheng Li, Department of Gastroenterology, The First Affiliated Hospital of Zhengzhou University, 1 Jianshe East Road, Zhengzhou, Henan 450052, P.R. China

E-mail: lijiansheng@medmail.com.cn

*Contributed equally

Abbreviations: HBV, hepatitis B virus; cccDNA, covalently closed circular DNA; CRISPR, clustered regularly interspaced short palindromic repeat; Cas9, CRISPR-associated protein 9; $\mathrm{PDH}$, primary duck hepatocyte; DHBV, duck hepatitis B virus; sgRNAs, single-guide RNAs; ETV, entecavir; NAs, nucleoside analogues; sgNS, one nonsense sgRNA; ZFN, zinc finger nucleases; TALEN, transcription activator-like effector nucleases

Key words: CRISPR/cas9, duck hepatitis B virus, hepatitis B virus, covalently closed circular DNA, antivirus polymerase chain reaction. The combined inhibition of CRISPR/Cas9 system and entecavir (ETV) was also assessed. Two sgRNAs, sgRNA4 and sgRNA6, exhibited efficient inhibition on DHBV total DNA (77.23 and $86.51 \%$, respectively), cccDNA (75.67 and 85.34\%, respectively) in PDHs, as well as DHBV total DNA in the culture medium (62.17 and 59.52\%, respectively). The inhibition remained or enhanced from day 5 to day 9 following transfection. The combination of the CRISPR/Cas9 system and ETV further increased the inhibitory effect on DHBV total DNA in PDHs and culture medium, but not cccDNA. The CRISPR/Cas9 system has the potential to be a useful tool for the suppression of DHBV DNA.

\section{Introduction}

Hepatitis B virus (HBV) infection remains a major public health problem worldwide at present. Patients with chronic $\mathrm{HBV}$ infection are at high risk of progressing to cirrhosis, hepatocellular carcinoma and liver failure. It is estimated that $\sim 800,000$ people die from HBV-related diseases per year (1). Although the introduction of the hepatitis $B$ vaccine into national immunization programs has dramatically reduced the incidence of $\mathrm{HBV}$ infection, the rate of vertical transmission, especially for hepatitis B virus e antigen-positive mothers, is up to $9.8 \%$ in China in 2002 (2). Nucleoside analogues (NAs), such as entecavir (ETV), lamivudine and adefovir dipivoxil can inhibit the reverse transcription of HBV mRNA and have been used as primary antiviral agents for the treatment of HBV infection in clinical practice. Nevertheless, they cannot radically eliminate HBV covalently closed circular (cccDNA) in the nucleus of hepatocytes, which is the template for HBV replication (3). Moreover, drug resistance may occur following long-term use of NAs. Thus, it is urgent to find out new efficient methods to eliminate HBV cccDNA.

The clustered regularly interspaced short palindromic repeat (CRISPR)/CRISPR-associated (Cas) system, originally identified in bacteria and archaea, is the third generation of genome-editing technology (4). The type II CRISPR/Cas system from Streptococcus pyogenes and its simplified derivative, the Cas9/single guide RNA (sgRNA) system $(5,6)$, has emerged as a potent new tool for targeted gene modification 
in humans and several other species (7-10). Since 2013, CRISPR/cas9-mediated genome editing has been successfully finished on several human viruses, such as papilloma virus, human immunodeficiency virus and Epstein-Barr virus (11-13).

So far, several reports have indicated that HBV total DNA and HBV cccDNA in infected hepatocytes can be reduced by the CRISPR/cas9 system (14-16). However, most of these studies have used HBV-infected human hepatoma cell lines, which are not considered as optimal cell models for human HBV infection, to evaluate drug efficacy. Therefore, it is essential to evaluate the inhibition efficacy of the CRISPR/Cas9 system in the setting of primary hepatocytes with natural HBV infection. As a model virus of HBV, duck hepatitis B virus (DHBV) shares the similar virus structure and replication features with HBV (17). In this respect, primary duck hepatocytes (PDHs) naturally infected with DHBV provide valuable model systems for studying HBV infection (18). In light of this, the authors hypothesized that the CRISPR/Cas9 system may suppress DHBV DNA. In order to validate the hypothesis, firstly, DHBV-specific sgRNA/Cas9 dual expression vector was constructed and transfected into DHBV-infected PDHs. Secondly, the inhibition efficacy on DHBV total DNA and cccDNA by the CRISPR/Cas9 system was evaluated. Finally, the combined inhibition of CRISPR/cas9 system and ETV was assessed.

\section{Materials and methods}

Design and construction of DHBV-specific sgRNA/Cas 9 plasmids. sgRNAs were designed using the CRISPR/Cas system (Cas9/gRNA) Off-Targeter (CasOT) tool (http://casot. cbi.pku.edu.cn/; Peking University, Beijing, China) to minimize potential off-target effects. The sequences of six sgRNAs targeting DHBV genome (GenBank: K01834.1) and one nonsense sgRNA (sgNS) are presented in Table I.

The PSpCas9(BB)-2A-GFP (PX458) plasmid was obtained from Addgene, Inc. (Cambridge, MA, USA). The plasmid was extracted using the EndoFree Mini Plasmid Kit II (Tiangen Biotech Co., Ltd., Beijing, China). PX458 was digested with BbsI (New England Biolabs, Inc., Ipswich, MA, USA), and then the linearized vector was purified using the Gel Extraction kit (Omega Bio-tek, Inc., Norcross, GA, USA) according to the manufacturer's instructions. Each pair of sgRNAs was annealed to double strands, which was ligated to the linearized vector with T4 DNA ligase (New England Biolabs, Inc.). The co-expression plasmid sgRNA/Cas9 was identified through sequencing (Fig. 1).

Isolation, infection and transfection of PDHs. Ducklings (1-day-old) were purchased from Qianjin Duck Farm (Beijing, China). All animal care and experimental procedures were performed with the approval of the Institutional Animal Care and Use Committee at Beijing Youan Hospital affiliated to Capital Medical University (Beijing, China) according to the Guide for the Care and Use of Laboratory Animals (National Institutes of Health, Bethesda, MD, USA). DHBV-positive and -negative ducklings were identified by polymerase chain reaction (PCR) using Ex Taq DNA polymerase (Takara Biotechnology Co., Ltd., Dalian, China) using serum samples. Sample processing, the corresponding primers (DHBV2548 and DHBV2840R), PCR reaction mixture and amplification cycle was described previously (18). The PCR product was verified by agarose gel electrophoresis (Fig. 2).

PDHs were isolated from 7-day-old DHBV-free Pekin ducklings (19). Isolated PDHs were seeded in a 24-well plate at $1.0 \times 10^{5} /$ well, and were cultured in L-15 medium (Invitrogen; Thermo Fisher Scientific, Inc., Waltham, MA, USA) supplemented with 10\% fetal bovine serum (Hyclone; GE Healthcare Life Sciences, Logan, UT, USA), 10 uM hydrocortisone (Sigma-Aldrich; Merck KGaA, Darmstadt, Germany), $10 \mu \mathrm{g} / \mathrm{ml}$ insulin (Cell Applications, Inc., San Diego, CA, USA), $20 \mathrm{mM}$ HEPES, and 1\% penicillin/streptomycin (both from Sigma-Aldrich; Merck KGaA) in a humidified chamber at $39^{\circ} \mathrm{C}$ without $\mathrm{CO}_{2}$. The next day, PDHs were infected with DHBV-positive serum $\left(\sim 4 \times 10^{6}\right.$ copies/well). On the third day following seeding, DHBV-infected PDHs were transfected with DHBV-specific sgRNA/Cas9 dual expression vector using Lipofectamine 2000 (Invitrogen; Thermo Fisher Scientific, Inc.). The ratio between Lipofectamine 2000 ( $\mu 1)$ and plasmid $(\mu \mathrm{g})$ was 3:1. Some wells were treated with ETV (China Food and Drug Administration, Beijing, China) at $0.13 \mathrm{nM}$ (20) on days 3,5, and 7 following transfection. The culture medium and cells were harvested on day 5 or day 9 following transfection for analyses.

Extraction of DHBV total DNA and cccDNA. DHBV total DNA in the culture medium was extracted using TIANamp Virus DNA/RNA kit (Tiangen Biotech Co., Ltd.). Following the removal of the culture medium, the cells were lysed and total DNA was extracted using TIANamp Genomic DNA kit (Tiangen Biotech Co., Ltd.). For the purification of DHBV cccDNA, DHBV total DNA was further treated with Plasmid-Safe $^{\mathrm{TM}}$ ATP-Dependent DNase (Epicenter; Illumina, Inc., San Diego, CA, USA) at $37^{\circ} \mathrm{C}$ for $30 \mathrm{~min}$ followed by $70^{\circ} \mathrm{C}$ for $30 \mathrm{~min}$ to digest linear double-stranded DNA, and the resulting product was recycled using Cycle Pure kit (Omega Bio-tek, Inc.) according to the instructions of manufacturer.

Quantification of DHBV DNA in the culture medium and $P D H s$. DHBV total DNA and cccDNA were then quantified by reverse transcription-quantitative PCR with specific primers and TaqMan MGB probes (Sangon Biotech Co., Ltd, Shanghai, China). The sequences of the primers and the corresponding TaqMan probes are displayed in Table II. PCR was performed using the StepOnePlus Real-time PCR system (Applied Biosystems; Thermo Fisher Scientific, Inc.). In a final volume of $20 \mu \mathrm{l}$, the following was added: $10 \mu \mathrm{l}$ SsoAdvanced ${ }^{\mathrm{TM}}$ Universal Probes SuperMix (Bio-Rad Laboratories, Inc., Hercules, CA, USA), $2 \mu$ l DNA template, $2 \mu 1$ forward and reverse mixed primers $(5 \mathrm{mM}), 1 \mu \mathrm{l}$ TaqMan probe $(2.5 \mathrm{mM})$ and $5 \mu \mathrm{l}$ double distilled water. Amplification was performed under the following conditions: $95^{\circ} \mathrm{C}$ for $30 \mathrm{sec}, 40$ cycles of $95^{\circ} \mathrm{C}$ for $5 \mathrm{sec}, 60^{\circ} \mathrm{C}$ for $30 \mathrm{sec}$, and $72^{\circ} \mathrm{C}$ for $30 \mathrm{sec}$. Duck $\beta$-globin gene was used as an internal reference. The relative quantification of total DNA and cccDNA was standardized to that of the sgNS group.

Statistical analysis. Normally distributed data were presented as mean \pm standard error of the mean and analyzed by Student's t-test. Non-normally distributed data were expressed as the median (range) and was analyzed by the Mann-Whitney U test. 
Table I. Sequence of the protospacer and protospacer adjacent motif targeted by DHBV-specific sgRNAs in the DHBV genome.

\begin{tabular}{|c|c|c|c|}
\hline Name & Nucleotide position & Gene region & Sequence $\left(\mathrm{GN}_{18-20} \mathrm{NGG}\right)$ \\
\hline sgRNA1 & $1,310-1,332$ & S & $\begin{array}{l}\text { F: 5'-GAGCTGGCCTAATCGGATTACTGG-3' } \\
\text { R: 5'-CCAGTAATCCGATTAGGCCAGCTC-3' }\end{array}$ \\
\hline sgRNA2 & $1,293-1,315$ & S & $\begin{array}{l}\text { F: 5'-GACCTTCGGGGGAATACTAGCTGG-3' } \\
\text { R: 5'-CCAGCTAGTATTCCCCCGAAGGTC-3' }\end{array}$ \\
\hline sgRNA3 & $1,363-1,385$ & S & $\begin{array}{l}\text { F: 5'-GAAATACTGAGGAGGCTAGATTGG-3' } \\
\text { R: 5'-CCAATCTAGCCTCCTCAGTATTTC-3' }\end{array}$ \\
\hline sgRNA4 & $1,449-1,472$ & $\mathrm{~S}$ & $\begin{array}{l}\text { F: 5'-GCAAATCTCTCCACATTACGTAGG-3' } \\
\text { R: 5'-CCTACGTAATGTGGAGAGATTTGC-3' }\end{array}$ \\
\hline sgRNA5 & $2,738-2,760$ & $\mathrm{C}$ & $\begin{array}{l}\text { F: 5'-GAAGACGCTTTAGAGCCTTATTGG-3' } \\
\text { R: 5'-CCAATAAGGCTCTAAAGCGTCTTC-3' }\end{array}$ \\
\hline sgRNA6 & $29-51$ & $\mathrm{P}$ & $\begin{array}{l}\text { F: 5'-GTTAACGAGGAATCACTGGATAGG-3' } \\
\text { R: 5'-CCTATCCAGTGATTCCTCGTTAAC-3' }\end{array}$ \\
\hline sgNS & & & $\begin{array}{l}\text { F: 5'-GAAATCCTGCAGAAAGACCTGG-3' } \\
\text { R: 5'-CCAGGTCTTTCTGCAGGATTTC-3' }\end{array}$ \\
\hline
\end{tabular}

sgRNA, single-guide RNA; DHBV, duck hepatitis B virus.

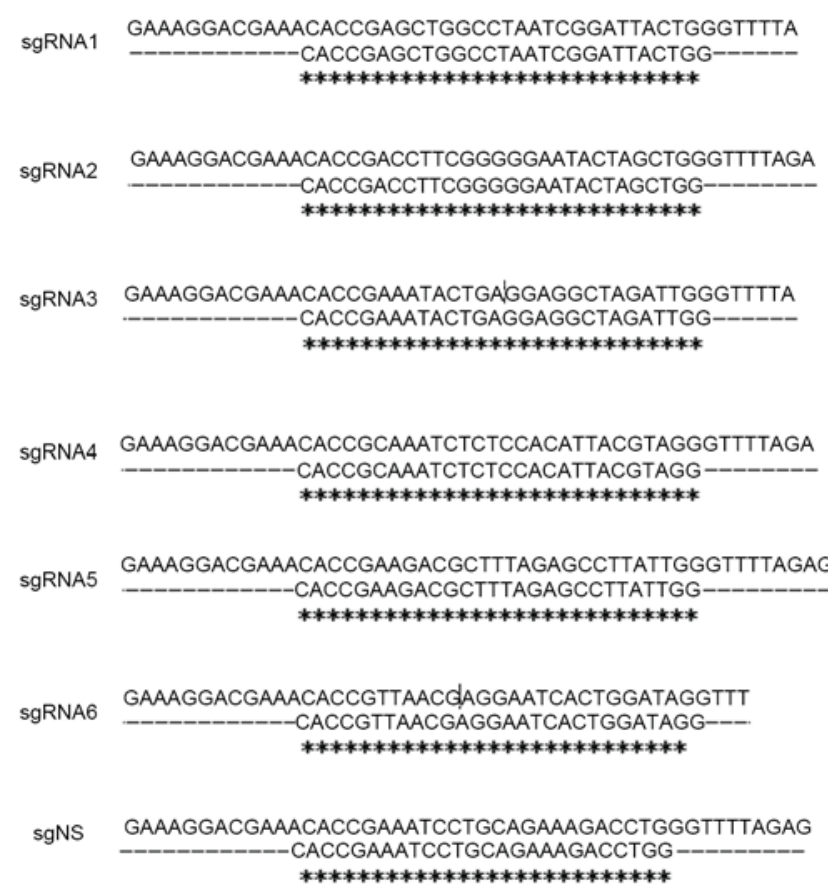

Figure 1. The constructed co-expression plasmids DHBV-specific sgRNA/Cas9 and sgNS/Cas9 were identified through sequencing. DHBV, duck hepatitis B virus; sgRNA, single-guide RNAs.

Statistical analysis was conducted using SPSS software (version, 19.0; IBM SPSS, Chicago, IL, USA). A two-sided $\mathrm{P}<0.05$ was considered to indicate a statistically significant difference.

\section{Results}

sgRNA4 and sgRNA6 significantly suppressed DHBV DNA on day 5 following transfection. To analyze the inhibition efficacy of six sgRNAs, the levels of DHBV DNA in PDHs were

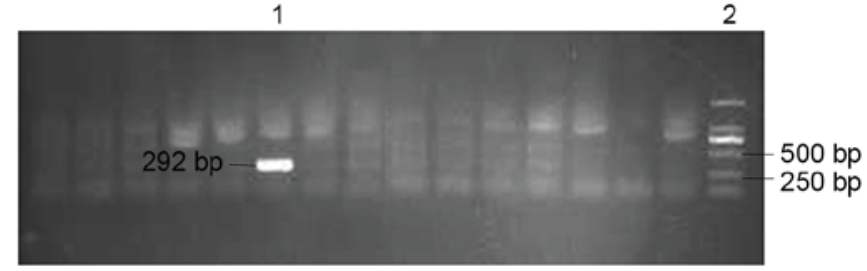

Figure 2. The identification of DHBV-positive serum by PCR. The size of the amplified PCR product was $292 \mathrm{bp}$. Lane 1, DHBV positive serum; and lane 2, DNA marker. DHBV, duck hepatitis B virus; PCR, polymerase chain reaction.

detected at various time points following transfection. Fig. 3 indicated that there was no significant difference among the three groups: No treatment group, Lipofectamine 2000 group and sgNS group. Compared with the sgNS group, sgRNA4 and sgRNA6 exhibited higher efficacy in suppressing DHBV DNA on day 5 following transfection. For sgRNA4, DHBV total DNA in PDHs was reduced by $77.64 \%$, (for example, from $1.30 \times 10^{3}$ copies/cell to $2.96 \times 10^{2}$ copies/cell). A similar reduction $(60.19 \%)$ was observed for sgRNA6. In addition, DHBV cccDNA was also suppressed significantly by sgRNA4 and sgRNA6 (60.19 and 68.82\%, respectively). The inhibition efficacy of sgRNA4 and sgRNA6 on DHBV total DNA and cccDNA presented a significant difference compared with $\operatorname{sgNS}(\mathrm{P}=0.002$ and 0.015 respectively for $\operatorname{sgRNA} 4, \mathrm{P}=0.003$ and 0.004 respectively for sgRNA6).

The inhibition efficacy of sgRNA4 and sgRNA6 remained or improved on day 9 following transfection. From day 5 to day 9 following transfection, the inhibition on DHBV total DNA remained (from 77.64 to $77.23 \%$ ) by sgRNA4, but increased (from 73.51 to $86.51 \%$ ) by sgRNA6. DHBV cccDNA in PDHs was further inhibited by sgRNA4 (from 60.19 to $75.67 \%$ ) and sgRNA6 (from 68.82 to $85.34 \%$ ). Moreover, DHBV total DNA 
Table II. DHBV primers for polymerase chain reaction.

\begin{tabular}{lll}
\hline Name & \multicolumn{1}{c}{ Primer } & Sequence $\left(5^{\prime}-3^{\prime}\right)$ \\
\hline cccDNA & Forward & TGCCATAAGCGTTATCAGACGTT \\
& Reverse & GGCTAAGGCTCTAGAAGCATTGA \\
Total DNA & TaqMan probe & ATATAATCCTGCTGACGGCC \\
& Forward & TTCGGAGCTGCTTGCCAA \\
& Reverse & TCATACACATTGGCTAAGGCTCT \\
& TaqMan probe & CGTCTACATTGCTGTTGTCGTGTGTGAC \\
& Forward & AGCAGTTGTTGGAGCAGGAA \\
& TaqMan probe & TCTTTGGCTGTTGGCATCTA \\
\end{tabular}

DHBV, duck hepatitis B virus; cccDNA, covalently closed circular DNA.
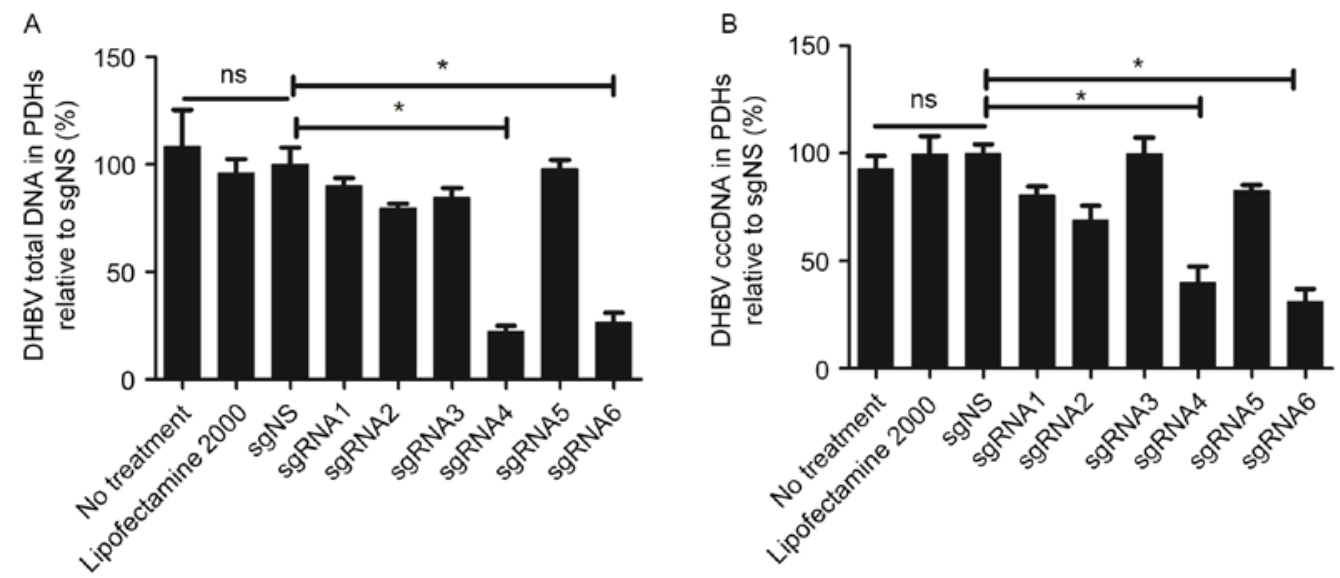

Figure 3. sgRNA4/Cas9 and sgRNA6/Cas9 efficiently inhibit the replication of DHBV on day 5 following transfection. The relative quantification of (A) total DNA and (B) cccDNA was standardized to that of the sgNS group. The percentages of DHBV total DNA and cccDNA expression were presented as mean \pm standard error of the mean. Comparisons were performed between the sgRNAs groups and the sgNS group. ${ }^{*} \mathrm{P}<0.05$. ns, no significant difference among the three groups: No treatment group, the Lipofectamine 2000 group and sgNS group. sgRNA, single-guide RNAs; Cas9, CRISPR-associated protein 9; DHBV, duck hepatitis B virus; sgNS, one nonsense sgRNA; cccDNA, covalently closed circular DNA; PDHs, primary duck hepatocytes.

in the culture medium was reduced by 62.17 and $59.52 \%$, respectively for sgRNA4 and sgRNA6 (Fig. 4).

ETV enhanced the suppression on DHBV DNA accumulation by CRISPR/Cas 9 system. Considering that the suppression on DHBV DNA by CRISPR/Cas9 system was incomplete, it would be interesting to further assess the combined inhibition of CRISPR/Cas9 system and ETV, the first-line treatment option for patients with HBV infection. As presented in Fig. 5, on day 9 following transfection (the sixth day following ETV treatment), the inhibition efficacy on DHBV total DNA in PDHs was higher in sgRNA4+ETV (97.52\%) and sgRNA6+ETV $(96.57 \%)$ groups compared with sgRNA4 (77.23\%) and sgRNA6 (86.51\%) groups $(\mathrm{P}=0.006$ and 0.005 respectively). Similarly, the inhibition efficacy on DHBV total DNA in the culture medium was higher in sgRNA4+ETV $(85.45 \%)$ and sgRNA6+ETV (78.29\%) groups compared with sgRNA4 (62.17\%) and sgRNA6 (59.52\%) groups $(\mathrm{P}=0.006$ and 0.011 , respectively). However, ETV treatment did not enhance the inhibition on DHBV cccDNA in PDHs by sgRNA4 (sgRNA4 vs. sgRNA4+ETV: $75.67 \%$ vs. $73.90 \%$; $\mathrm{P}=0.144$ ) and sgRNA6 (sgRNA6 vs. sgRNA6+ETV: $85.34 \%$ vs. 82.60\%; $\mathrm{P}=0.144$ ). Thus, the combination of ETV and CRISPR/Cas9 system led to a further reduction of DHBV total DNA in PDHs and culture medium, but not DHBV cccDNA.

\section{Discussion}

In the present study, two sgRNAs (sgRNA4 and sgRNA6) targeting the DHBV genome were demonstrated to suppress DHBV total DNA and cccDNA successfully. ETV enhanced the inhibition of the CRISPR/Cas9 system on DHBV total DNA. The current findings suggested that the CRISPR/Cas9 system targeting specific sites of the DHBV genome may be an effective technology to inhibit DHBV infection in PDHs. To the best of the authors' knowledge, the present study is the first reporting the targeted inhibition of DHBV DNA by the CRISPR/Cas9 system.

Genetic modifications have been achieved successfully using genome-editing technologies, including zinc finger nucleases (ZFN) (21), transcription activator-like effector nucleases (TALEN) (22) and the CRISPR/Cas9 system (23). 
A

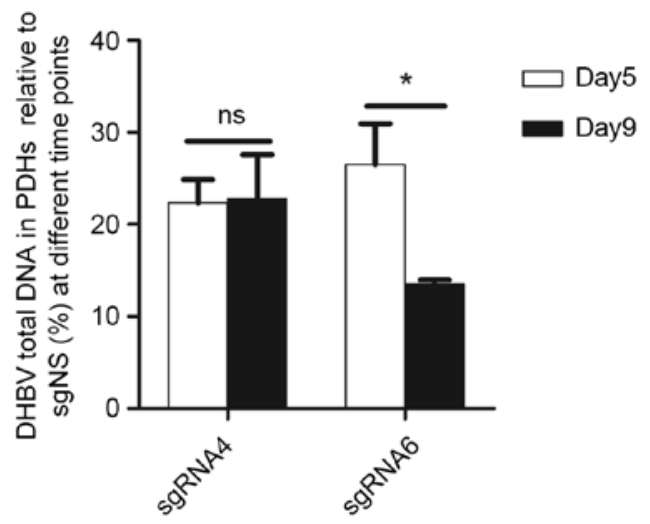

B

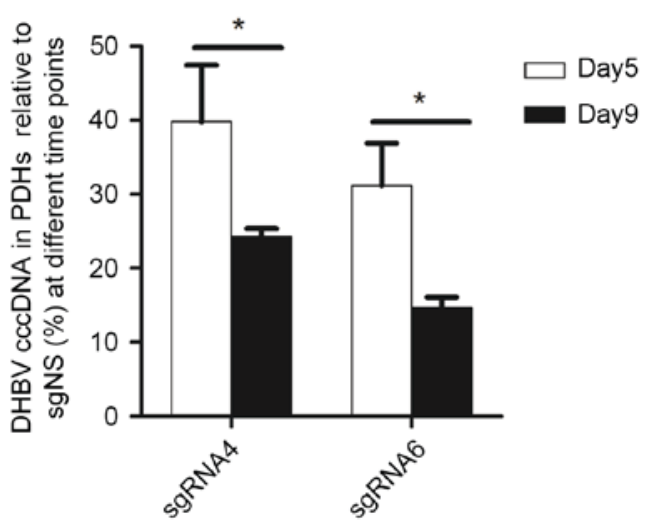

Figure 4. The inhibition on DHBV DNA, especially cccDNA, increases from day 5 to day 9 following transfection. The levels of DHBV (A) total DNA and (B) cccDNA on day 5 and day 9 following transfection were compared. " $\mathrm{P}<0.05$. ns, no significant difference between the two groups. DHBV, duck hepatitis B virus; cccDNA, covalently closed circular DNA; PDHs, primary duck hepatocytes.
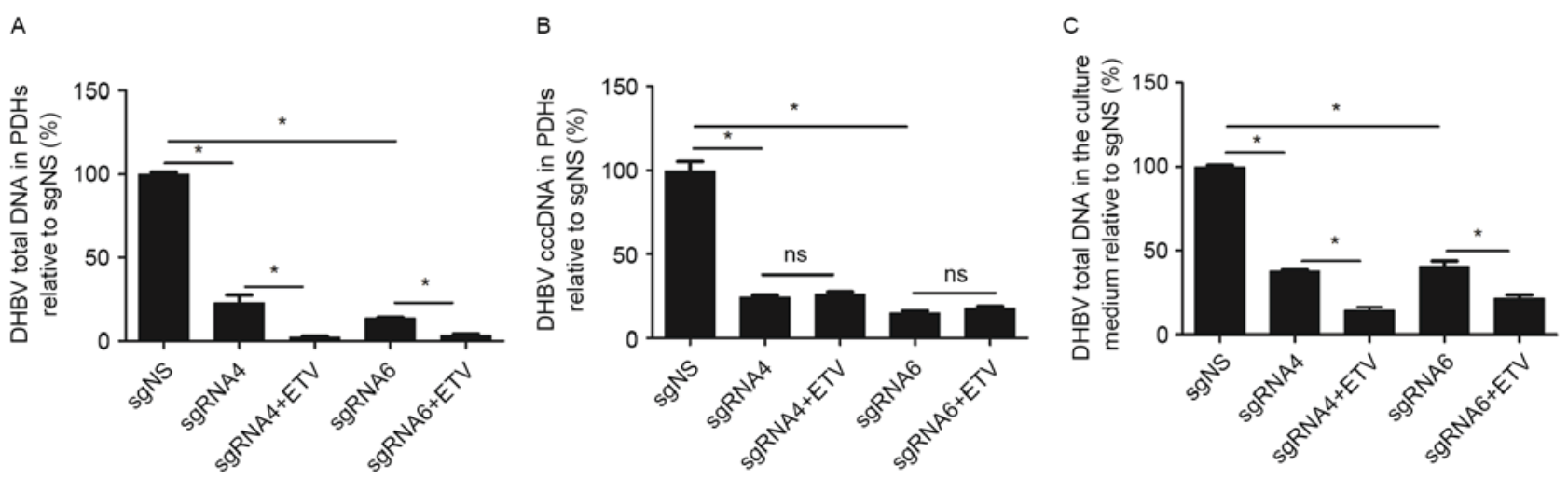

Figure 5. ETV enhances the inhibition of sgRNA4 and sgRNA6 on DHBV total DNA, but not cccDNA. On the day 9 following transfection, DHBV (A) total DNA in PDHs and (B) culture medium as well as (C) cccDNA in PDHs were measured by reverse transcription-quantitative polymerase chain reaction. The relative quantification of total DNA and cccDNA was standardized to that of the sgNS group. The percentages of total DNA and cccDNA expression were presented as mean \pm standard error of the mean. The statistical analysis was performed between the sgRNA groups and the sgNS group. ${ }^{*} \mathrm{P}<0.05$. ns, no significant difference between the two groups. ETV, entecavir; sgRNA, single-guide RNAs; DHBV, duck hepatitis B virus; PDHs, primary duck hepatocytes; ccDNA, covalently closed circular DNA; sgNS, one nonsense sgRNA.

As the classical method for genome engineering, $\mathrm{ZFN}$ is used to invalidate the HIV co-receptor $\mathrm{C}-\mathrm{C}$ chemokine receptor type 5, which is currently in clinical trials (NCT01252641, NCT00842634 and NCT01044654). It is reported that TALEN plasmids for 18,740 human protein-coding genes have been assembled (24). However, both ZFNs and TALENs utilize protein-based programmable, sequence-specific DNA-binding modules, whose construction is usually complex. The emergence of the CRISPR/Cas9 system in 2013 (25) makes it a facile and efficient alternative to ZFNs and TALENs. Only sgRNAs targeting specific genes are required for highly efficient gene modification using the CRISPR/Cas9 system $(26,27)$.

In the present study, the effective sgRNAs targeting the DHBV genome were located at the $\mathrm{S}$ and $\mathrm{P}$ regions. Previous studies also screened out effective sgRNAs located at different regions of $\mathrm{HBV}$ genome, such as $\mathrm{X}$, core, polymerase and surface ORFs $(14,15,28)$. sgRNAs targeting the conserved HBV sequence were effective for HBV genomes of different genotypes (29). Thus, it may be necessary to construct the sgRNAs library targeting different regions of HBV genome in order to screen out the most efficient sgRNAs.
Several previous reports used either Huh7 cells (29) transfected with the HBV-expression vector pAAV/HBV1.2 (genotype A) or HepAD38 cells (30) stably expressing HBV DNA to evaluate the targeted inhibition on HBV genome by the CRISPR/Cas9 system. In terms of biological characteristics and HBV infection mode, PDHs infected with DHBV-positive serum have advantages over immortalized cell lines, because the former may mimic the natural process of $\mathrm{HBV}$ infection. In the present study, we chose DHBV-infected PDHs as an in vitro model to assess the inhibition efficiency of the CRISPR/Cas9 system. In this regard, the result that the CRISPR/Cas9 system can efficiently inhibit DHBV cccDNA is more close to the real-world $\mathrm{HBV}$ infection, thus exhibiting great clinical importance. Moreover, the study investigated whether ETV can improve the anti-viral effects of CRISPR/Cas9 system. The results indicated that the combination of the CRISPR/Cas9 system and ETV enhanced the suppression of DHBV total DNA, but not cccDNA. One possible explanation is that ETV, as a nucleoside reverse transcriptase inhibitor, could only reduce DHBV total DNA, but has little or no effect on DHBV cccDNA. Therefore, it was speculated that the combined 
application of the CRISPR/Cas9 system and ETV may have the potential to control DHBV infection more effectively.

There are two limitations in the present study. Firstly, sgRNAs targeting different regions of DHBV genome need to be designed in order to screen out the most effective ones. Secondly, in vivo studies need to be performed to validate the inhibitory effect of the CRISPR/Cas9 system on DHBV cccDNA.

Although great advancements have been made in the prevention and treatment of HBV infection, the high morbidity of HBV-associated complications is still a huge threat for human health. The present study is thought to be the first to demonstrate the efficient inhibition of the CRISPR/Cas9 system on DHBV cccDNA. Even though further study is required to improve the inhibitory efficiency, the current findings pave the way for eliminating HBV cccDNA using the CRISPR/Cas9 system in clinical practice in the future.

\section{Acknowledgements}

The present study was supported by the National Science and Technology Key Project (grant nos. 2017ZX10201201, 2017ZX10203201-005, 2017ZX10202203-006-001 and 2017ZX10302201-004-002), the Beijing Municipal Administration of Hospital's Ascent Plan (grant no. DFL20151601), the Beijing Municipal Science and Technology Commission (grant no. Z151100004015066), the Basic-Clinical Cooperation Project of Capital Medical University (grant nos. 15JL67 and 17JL47) and the YouAn Fund for Liver Diseases and AIDS (grant no. YNKT20160012).

\section{References}

1. Komatsu H: Hepatitis B virus: Where do we stand and what is the next step for eradication? World J Gastroenterol: 20: 8998-9016, 2014.

2. Xu DZ, Yan YP, Choi BC, Xu JQ, Men K, Zhang JX, Liu ZH and Wang FS: Risk factors and mechanism of transplacental transmission of hepatitis B virus: A case-control study. J Med Virol 67: 20-26, 2002.

3. Werle-Lapostolle B, Bowden S, Locarnini S, Wursthorn K, Petersen J, Lau G, Trepo C, Marcellin P, Goodman Z Delaney WE VI, et al: Persistence of cccDNA during the natural history of chronic hepatitis B and decline during adefovir dipivoxil therapy. Gastroenterology 126: 1750-1758, 2004.

4. Hsu PD, Lander ES and Zhang F: Development and applications of CRISPR-Cas9 for genome engineering. Cell 157: 1262-1278, 2014.

5. Barrangou R and Marraffini LA: CRISPR-Cas systems: Prokaryotes upgrade to adaptive immunity. Mol Cell 54: 234-244, 2014.

6. Cong L, Ran FA, Cox D, Lin S, Barretto R, Habib N, Hsu PD, Wu X, Jiang W, Marraffini LA and Zhang F: Multiplex genome engineering using CRISPR/Cas systems. Science 339: 819-823, 2013.

7. Wang T, Wei JJ, Sabatini DM and Lander ES: Genetic screens in human cells using the CRISPR-Cas9 system. Science 343: 80-84, 2014.

8. Yang H, Wang H, Shivalila CS, Cheng AW, Shi L and Jaenisch R: One-step generation of mice carrying reporter and conditional alleles by CRISPR/Cas-mediated genome engineering. Cell 154: $1370-1379,2013$

9. Gratz SJ, Cummings AM, Nguyen JN, Hamm DC, Donohue LK, Harrison MM, Wildonger J and O'Connor-Giles KM: Genome engineering of Drosophila with the CRISPR RNA-guided Cas9 nuclease. Genetics 194: 1029-1035, 2013.

10. Chang N, Sun C, Gao L, Zhu D, Xu X, Zhu X, Xiong JW and Xi JJ: Genome editing with RNA-guided Cas9 nuclease in zebrafish embryos. Cell Res 23: 465-472, 2013.
11. Hu Z, Yu L, Zhu D, Ding W, Wang X, Zhang C, Wang L, Jiang X, Shen H, He D et al: Disruption of HPV16-E7 by CRISPR/Cas system induces apoptosis and growth inhibition in HPV16 positive human cervical cancer cells. Biomed Res Int 2014: 612823 , 2014.

12. Hu W, Kaminski R, Yang F, Zhang Y, Cosentino L, Li F, Luo B, Alvarez-Carbonell D, Garcia-Mesa Y, Karn J, et al: RNA-directed gene editing specifically eradicates latent and prevents new HIV-1 infection. Proc Natl Acad Sci USA 111: 11461-11466, 2014

13. Wang J and Quake SR: RNA-guided endonuclease provides a therapeutic strategy to cure latent herpesviridae infection. Proc Natl Acad Sci USA 111: 13157-13162, 2014.

14. Dong C, Qu L, Wang H, Wei L, Dong Y and Xiong S: Targeting hepatitis B virus cccDNA by CRISPR/Cas9 nuclease efficiently inhibits viral replication. Antiviral Res 118: 110-117, 2015.

15. Seeger C and Sohn JA: Targeting hepatitis B virus with CRISPR/Cas9. Mol Ther Nucleic Acids 3: e216, 2014.

16. Zhen S, Hua L, Liu YH, Gao LC, Fu J, Wan DY, Dong LH, Song HF and Gao X: Harnessing the clustered regularly interspaced short palindromic repeat (CRISPR)/CRISPR-associated Cas 9 system to disrupt the hepatitis B virus. Gene Ther 22: 404-412, 2015.

17. Jilbert AR, Botten JA, Miller DS, Bertram EM, Hall PM, Kotlarski J and Burrell CJ: Characterization of age- and dose-related outcomes of duck hepatitis B virus infection. Virology 244: 273-282, 1998.

18. Zhang YY, Theele DP and Summers J: Age-related differences in amplification of covalently closed circular DNA at early times after duck hepatitis B virus infection of ducks. J Virol 79: 9896-9903, 2005.

19. Tuttleman JS, Pugh JC and Summers JW: In vitro experimental infection of primary duck hepatocyte cultures with duck hepatitis B virus. J Virol 58: 17-25, 1986.

20. Marion PL, Salazar FH, Winters MA and Colonno RJ: Potent efficacy of entecavir (BMS-200475) in a duck model of hepatitis $\mathrm{B}$ virus replication. Antimicrob Agents Chemother 46: 82-88, 2002.

21. Miller JC, Holmes MC, Wang J, Guschin DY, Lee YL, Rupniewski I, Beausejour CM, Waite AJ, Wang NS, Kim KA, et al: An improved zinc-finger nuclease architecture for highly specific genome editing. Nat Biotechnol 25: 778-785, 2007.

22. Miller JC, Tan S, Qiao G, Barlow KA, Wang J, Xia DF, Meng X, Paschon DE, Leung E, Hinkley SJ, et al: A TALE nuclease architecture for efficient genome editing. Nat Biotechnol 29: 143-148, 2011.

23. Barrangou R: RNA-mediated programmable DNA cleavage. Nat Biotechnol 30: 836-838, 2012.

24. Kim Y, Kweon J, Kim A, Chon JK, Yoo JY, Kim HJ, Kim S, Lee C, Jeong E, Chung E, et al: A library of TAL effector nucleases spanning the human genome. Nat Biotechnol 31: 251-258, 2013.

25. Grissa I, Vergnaud G and Pourcel C: The CRISPRdb database and tools to display CRISPRs and to generate dictionaries of spacers and repeats. BMC Bioinformatics 8: 172, 2007.

26. Ramanan V, Shlomai A, Cox DB, Schwartz RE, Michailidis E, Bhatta A, Scott DA, Zhang F, Rice CM, Bhatia SN, et al: CRISPR/Cas9 cleavage of viral DNA efficiently suppresses hepatitis B virus. Sci Rep 5: 10833, 2015.

27. Ebina H, Misawa N, Kanemura Y and Koyanagi Y: Harnessing the CRISPR/Cas9 system to disrupt latent HIV-1 provirus. Sci Rep 3: 2510, 2013.

28. Kennedy EM, Kornepati AV and Cullen BR: Targeting hepatitis B virus cccDNA using CRISPR/Cas9. Antiviral Res 123: 188-192, 2015.

29. Lin SR, Yang HC, Kuo YT, Liu CJ, Yang TY, Sung KC, Lin YY, Wang HY, Wang CC, Shen YC, et al: The CRISPR/Cas9 system facilitates clearance of the intrahepatic HBV templates In Vivo. Mol Ther Nucleic Acids 3: e186, 2014.

30. Kennedy EM, Bassit LC, Mueller H, Kornepati AV, Bogerd HP, Nie T, Chatterjee P, Javanbakht H, Schinazi RF, Cullen BR, et al: Suppression of hepatitis B virus DNA accumulation in chronically infected cells using a bacterial CRISPR/Cas RNA-guided DNA endonuclease. Virology 476: 196-205, 2015. 